\title{
Toroidal Field Reversals
}

\author{
T. M. Rogers ${ }^{1}$ \\ ${ }^{1}$ Department of Planetary Sciences, The University of Arizona \\ 1629 E. University Blvd. Tucson AZ 85721 \\ email: tami@lpl.arizona.edu
}

\begin{abstract}
I present axisymmetric numerical simulations of the solar interior, with differential rotation imposed in the convection zone and tachocline and a dipolar poloidal field confined to the radiative interior. In these simulations toroidal field reversals which are equator-ward propagating are driven in the absence of a dynamo. These reversals are driven in the tachocline and are seen at the top of the convection zone. While not solar-like in many ways, these reversals do show some solar-like properties not previously seen in full MHD simulations.
\end{abstract}

Keywords. Sun: interior, magnetic fields

\section{Introduction}

One of the main constraints on models and simulations of the solar dynamo process is the sunspot cycle, marked by equator-ward propagation of sunspot pairs. The basic picture of the sunspot cycle is that magnetic field is pumped into the tachocline by overshooting convection, where it is stretched and amplified by the strong radial and latitudinal differential rotation there. The toroidal field, thus amplified and strong enough to traverse the convection zone without being destroyed, becomes magnetically buoyant and rises through the convection zone, manifesting itself at the surface of the Sun as sunspot pairs. In flux-transport dynamo models the toroidal field is advected equatorward by the meridional circulation at the base of the convection zone. Of course, there are many uncertainties in this picture of the solar dynamo, including how well the field is pumped, organized, amplified in the tachocline, as well as how it becomes buoyant, remains in tact in its traverse through the convection zone, etc. Dynamo models usually focus on addressing the gross features of the sunspot cycle, such as the equator-ward propagation of toroidal field which reverses in time. Such solutions are easy to achieve in mean-field models, but have proven more difficult in more physical, less-tunable, magnetohydrodynamic (MHD) simulations of the solar convection zone. Simulations of dynamos in the solar convection zone from two decades ago (Glatzmaier (1985)) and more recent simulations (Brown (2010), Ghizaru et. al. (2010)) have produced reversing toroidal field but with generally pole-ward or no latitudinal propagation.

It was speculated early on (Glatzmaier (1985)) that equator-ward propagation could be achieved in the adjacent stable region, if the reversals and propagation were due to a dynamo wave (Parker (1955)) and obeyed the Parker-Yoshimura sign rule. Unfortunately, three dimensional calculations including an adjacent stable region are not abundant. The most recent of such simulations (Browning et. al. (2006)) showed that magnetic field was easily stored and amplified in an imposed tachocline, but saw no reversals. Here I show here that toroidal field reversals can be instigated by the Tayler instability and that equator-ward propagation occurs by advection of the toroidal field by meridional circulation, similar to flux-transport models. 


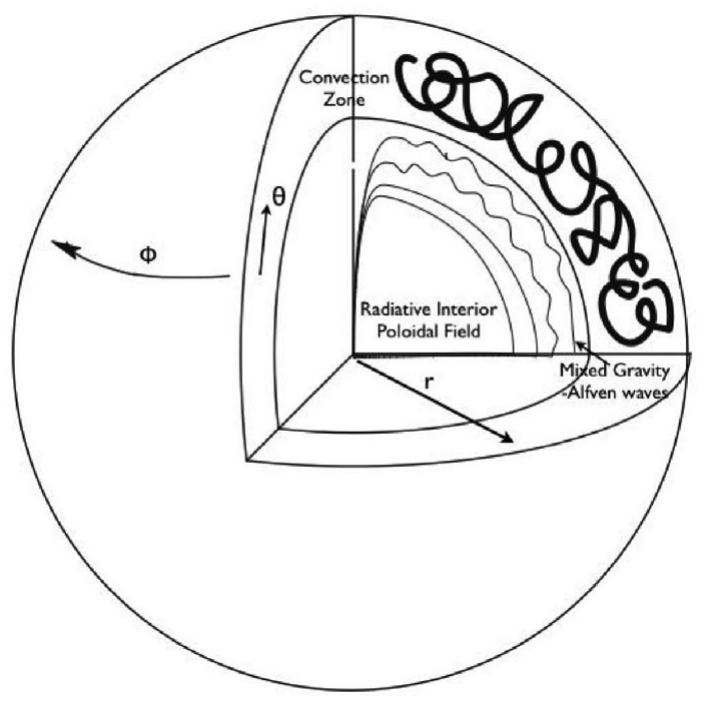

Figure 1. Schematic of model setup.

\section{Model Setup}

I emphasize that the simulations presented here are axisymmetric and therefore, not dynamo simulations. The original purpose of the simulations was to study the uniform rotation of the solar radiative interior $\dagger$. For that reason the models simulate a large fraction of the solar radius extending from $0.10 R_{\odot}$ to $0.90 R_{\odot}$ and accurately represents the stable stratification of the radiation zone (in order to better represent internal gravity waves). Because the model is axisymmetric I am not able to accurately simulate the differential rotation of the convection zone. Therefore, I artificially impose the observed differential rotation profile of the convection zone. After running a purely hydrodynamic model for some time I add a dipolar poloidal field to the radiative interior. The initial field configuration is such that the field lines close within the radiative interior but overlap the tachocline. A schematic of the model setup is shown in Figure $1 . \ddagger$

I solve the full MHD equations in the anelastic approximation:

$$
\begin{aligned}
\nabla \cdot \mathbf{B}= & 0 \\
\nabla \cdot(\bar{\rho} \mathbf{u})= & 0 \\
\frac{\partial \mathbf{u}}{\partial t}+(\mathbf{u} \cdot \nabla) \mathbf{u}= & -\nabla P-C \bar{g} \hat{r}+2(\mathbf{u} \times \Omega)+\frac{1}{\rho}(\mathbf{J} \times \mathbf{B}) \\
& +\bar{\nu}\left(\nabla^{2} \mathbf{u}+\frac{1}{3} \nabla(\nabla \cdot \mathbf{u})\right) \\
\frac{\partial T}{\partial t}+(\mathbf{v} \cdot \nabla) T= & -v_{r}\left(\frac{d \bar{T}}{d r}-(\gamma-1) \bar{T} h_{\rho}\right) \\
& +(\gamma-1) T h_{\rho} v_{r}+\gamma \bar{\kappa}\left[\nabla^{2} T+\left(h_{\rho}+h_{\kappa}\right) \frac{\partial T}{\partial r}\right]
\end{aligned}
$$

$\dagger$ Which I did discuss at the meeting but is not included in this proceeding

$\ddagger$ Since the time of the meeting this work has been submitted to The Astrophysical Journal, figures in this conference proceeding are the same as some of those depicted in that paper. 


$$
\frac{\partial \mathbf{B}}{\partial t}=\nabla \times(\mathbf{u} \times \mathbf{B})+\eta \nabla^{2} \mathbf{B}
$$

where equations 1 and 2 ensure magnetic flux and mass conservation. Equation 3 is the momentum equation, 4 is the energy equation and 5 is the magnetic induction equation. The numerical method is similar to that in Glatzmaier (1984), except here we use a finite difference scheme in the radial direction as opposed a Chebyshev expansion. This allows us more flexibility in allocation of radial resolution. The resolution of the model presented is 1500 radial zones by 512 latitudinal zones. In radius, 500 zones are devoted to the radiative interior and 1000 are devoted to the tachocline and convection zone.

\section{Results}

The model is initiated with a purely poloidal magnetic field. Toroidal field is quickly generated by stretching of that field by the differential rotation in the tachocline. The induction of toroidal field is described by the toroidal component of the induction equation, given by:

$\frac{\partial B_{\phi}}{\partial t}=r B_{r} \frac{\partial}{\partial r}\left(\frac{u_{\phi}}{r}\right)-r u_{r} \frac{\partial}{\partial r}\left(\frac{B_{\phi}}{r}\right)+\frac{\sin \theta B_{\theta}}{r} \frac{\partial}{\partial \theta}\left(\frac{u_{\phi}}{\sin \theta}\right)-\frac{\sin \theta u_{\theta}}{r} \frac{\partial}{\partial \theta}\left(\frac{B_{\phi}}{\sin \theta}\right)+\eta \nabla^{2} B_{\phi}$

Initially, toroidal field induction is dominated by the first term on the right hand side of equation 3.1 and as such the toroidal field is oppositely signed at high and low latitudes,

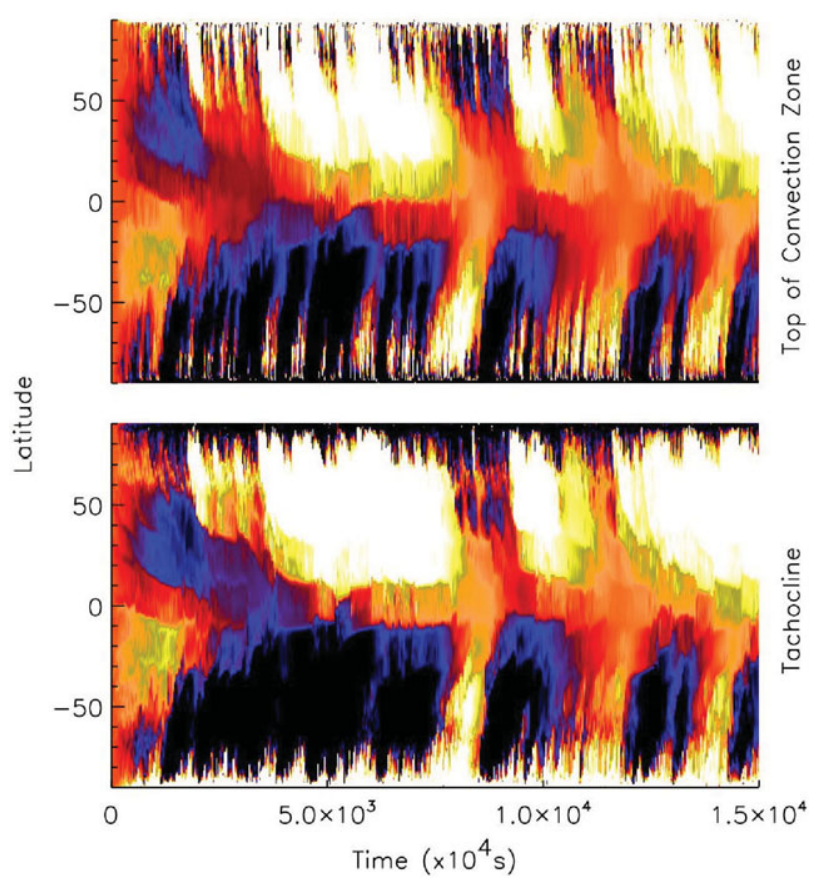

Figure 2. Toroidal field as a function of time and latitude. Blue (black) represents negative toroidal field, while white represents positive. The top panel shows the field at the top of the convection zone, while the bottom panel shows the field in the tachocline. 


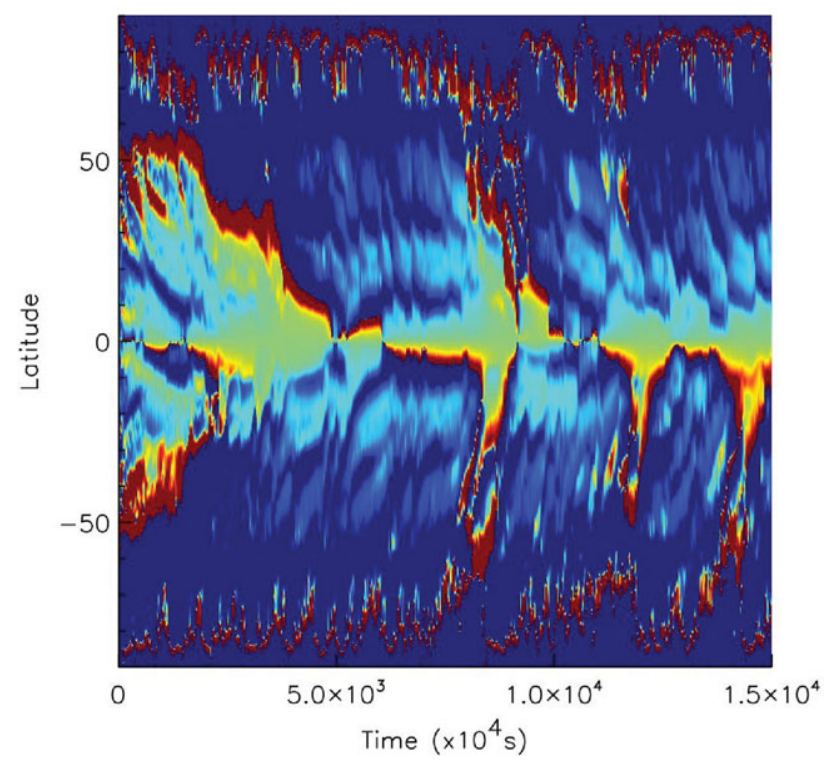

Figure 3. Tayler instability criterion, as described by equation 3.2 , in the tachocline, as a function of time and latitude. Blue represents times and regions where the model is stable, Red represents times and positions of instability. Instability clearly corresponds to times of "reversals" as seen in figure 2 .

anti-symmetric about the equator. This configuration quickly becomes unstable, leaving one dominant sign in the northern hemisphere and the opposite sign in the southern hemisphere (the sign dictated by the third term on the rhs of 3.1 and the initial poloidal field configuration). Throughout most of the simulation we find that the dominant induction term is the advection of toroidal field by latitudinal velocity (the fourth term on the rhs of 3.1). The dominant sign in each hemisphere remains fixed in time in the radiative interior. However, the sign of the toroidal field in the tachocline and convection zone changes in time, as can be seen in Figure 2. Clearly, there is a dominant toroidal field sign in each hemisphere (which I will refer to as the dominant sign) and a weaker field sign in each hemisphere (which I refer to as the minority sign). In the northern hemisphere, positive toroidal field is the dominant sign; in the southern hemisphere negative toroidal field is the dominant sign. Occasionally, the dominant sign toroidal field decays rapidly and the minority sign becomes dominant, leading to the appearance of a reversal. The cause of rapid toroidal field decay can be traced to the axisymmetric Tayler instability (Tayler (1975), Spruit (1999)). The basic criterion for the this instability (Spruit (1999)), is given by:

$$
\cos \theta \frac{\partial}{\partial \theta}\left(\ln \left(\frac{B^{2}}{\sin ^{2} \theta}\right)\right)>0
$$

In Figure 3 we show the lhs of equation 3.2 as a function of time and latitude at a height within the tachocline, with red (white) representing values larger than zero and blue (black) representing values less than zero. One can clearly see that reversals seen in Figure 2 are associated with times when the axisymmetric Tayler instability criterion is satisfied. In Figure 3 one can see that the criterion is satisfied much of the time at high latitudes, but reversals coincide with those times when the criterion is satisfied at low latitudes as well. 

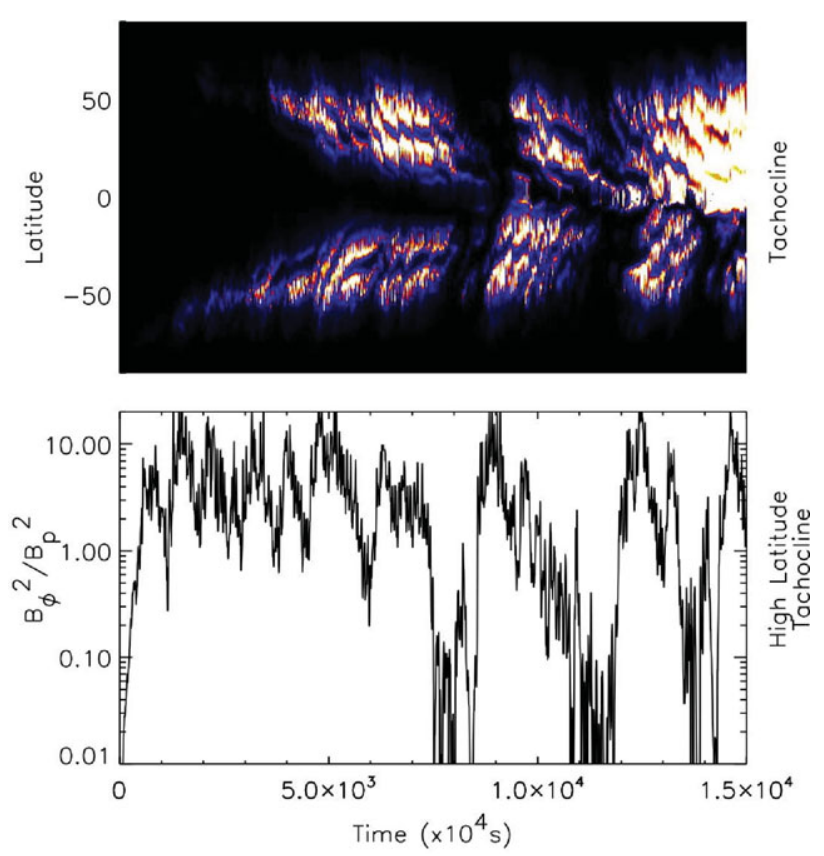

Figure 4. Ratio of toroidal to poloidal field strength, in the tachocline, as a function if time and latitude (top) and at high latitudes (bottom). Note that this ratio is larger at low latitudes due to the fact that the poloidal field is much larger at high latitudes than at low latitudes.

The condition in 3.2 is for a purely toroidal field and as shown by Wright (1973) and Braithwaite \& Spruit (2004), the addition of a poloidal field can have a stabilizing effect. Both showed that with mixed field components the instability criterion then also depends on the relative strengths and geometries of the toroidal and poloidal field components. I similarly find that the toroidal field must satisfy the gradient condition expressed in 3.2, but in addition, its strength must also overcome the stabilizing effect of the poloidal field, which occurs when the local ratio $B_{\phi}^{2} / B_{p}^{2}$ is $\approx 10-20$. Once these criterion are satisfied the toroidal field rapidly decays, as seen in Figure 4 . In Figure 4 I show the ratio of the toroidal field energy to the poloidal field energy as a function of time and latitude (top panel), while the bottom panel just shows this ratio at a chosen (high) latitude. Two things are immediately obvious in this figure. First, it is clear in the top panel of figure 4 that the ratio of field energy, $B_{\phi}^{2} / B_{p}^{2}$, is significantly higher at low latitudes than at high latitudes, which is predominantly due to the poloidal field in the tachocline being substantially larger at high latitudes than at low latitudes. $\dagger$ Second, the ratio of toroidal to poloidal magnetic energy is rather oscillatory at high latitudes, and occasionally after reaching a strong peak, there is rapid destruction of toroidal field energy. During these times, the Tayler instability criterion as described in 3.2, is satisfied and the minority signed toroidal field is advected equator-ward by meridional circulation. Looking at Figure 2 one can estimate the timescale for the minority field to be swept to the equator. Comparing this to the advection time calculated using the mean latitudinal velocity at the base of the convection zone $\left(\approx 3 \times 10^{3} \mathrm{~cm} / \mathrm{s}\right)$ one finds that the advection time is similar, confirming the dominance of the advection of toroidal field by latitudinal flow. Initially

$\dagger$ This implies that the field is not as well confined at high latitudes than at low latitudes, which has some implications for the ability of field to enforce uniform rotation in the solar interior. This matter will be discussed in a forthcoming paper. 


\section{Implications}

These simulations are clearly not good solar analogues. The short comings of this model are many: axisymmetry (lack of dynamo), artificially imposed differential rotation and large diffusion coefficients are the first to come to mind (there are others). Nevertheless, there are some interesting features. First, this model is the first MHD simulation which gets equator-ward propagating and reversing toroidal field, and does so without a dynamo. Second, this model demonstrates that the equator-ward propagation can easily be obtained by meridional circulation which is driven by the differential rotation in the solar convection zone, a key component of flux-transport mean field models. Finally, these simulations give another measure of stability for mixed field configurations.

\section{References}

Braithwaite, J. \& Spruit, H. C. 2007, ApJ, 469, 275

Brown, B. P., Browning, M. K., Brun, A. S., Miesch, M., \& Toomre, J. 2010, ApJ, 711, 424

Browning, M. K., Miesch, M., Brun, A. S., \& Toomre, J. 2006, ApJ, 648, L157

Ghizaru, M., Charbonneau, P., \& Smolarkiewicz, P. K. 2010, ApJL, 715, L133

Glatzmaier, G. 1985, GAFD, 30, 490

Glatzmaier, G. 1985, Journal of Computational Physics, 55, 461

Parker, E. N. 1955, ApJ, 122, 293

Spruit, H. C. 1999, A\&SA, 349,189

Tayler, R. J. 1975, Bulletin of the American Astronomical Society, 7,252

Wright, G. A. E. 1973, MNRAS, 162,339

\section{Discussion}

BRANDENBERG: How do you model the radiation conductivity? Did you use a rescaled Kramers opacity?

Rogers: The thermal diffusivity is modeled as the solar radiative conductivity, divided by $\rho c_{p}$, multiplied by some factor for numerical stability.

$$
\kappa(r)=f \frac{16 \sigma T^{3}}{3 \rho^{2} \chi c_{p}}
$$

where $\sigma$ is the Stefan-Boltzmann constant, and $\chi$ is the opacity.

BRANDENBERG: How realistic is the overshoot you get?

Rogers: I don't know if we know what "realistic" overshoot is, given we don't have a direct measurement. I think these are some of the best simulations of overshoot in the Sun since they have the stable stratification right and the gradient of the thermal diffusivity is right. The overshoot I get is very small, maybe $10 \%$ of a pressure scale height, depending on how it is defined.

Brandenberg: Do the g-modes restrict the timestep, i.e. how useful is it to use the anelastic approximation?

RoGERS: Yes, the gravity waves restrict the timestep, or probably more accurately the timestep can be limited by the angular velocity generated by the dissipation of gravity waves. This is particularly true at the center of the simulations where a small amount of angular momentum deposition could lead to somewhat large angular velocities compounded by the fact that the horizontal grid spacing there is very fine. 
BRUN: What is the main angular momentum transport process at the base of the convection zone in your simulation?

RoGERS: Note to reader: this question refers to the part of the talk where I discussed angular momentum transport in the radiative interior. This part of the talk is not included in this conference proceeding. I don't really know the answer to this question. Just below the convection zone the angular momentum transport is dominated by overshoot and waves, although referring to them as waves in this region is probably not accurate. In the models I have run the magnetic field has little influence in that region

BRun: How does the presence of magnetic field change the balance of angular momentum and the properties of the internal waves (propagation, group velocity...)?

RoGERS: In the axisymmetric models presented here the answer is I don't know. However, in models and simulations of IGW interacting with a purely toroidal field the answer is that magnetic fields can reflect the waves creating a duct and enhanced angular momentum deposition in the region between the field and the base of the convection zone. The magnetic field then filters some waves, preventing them from reaching the deep interior, but also acts as an amplification mechanism on other waves. The net is that the field causes the wave transport in the deep radiative interior to be highly dependent in time and space, because the reflection/amplification depends on the local field strength (which varies in time). 